\title{
Carryover of a saddle-node bifurcation after transforming a parameter into a variable
}

Carlos Contreras ( $\nabla$ carlos.contreras@ualberta.ca)

University of Alberta https://orcid.org/0000-0001-7928-607X

\section{Gustavo Carrero}

Athabasca University

Gerda de Vries

University of Alberta

\section{Research Article}

Keywords: Bifurcation, saddle-node bifurcation, bifurcation diagram, parameter transformation, mathematical modeling

Posted Date: August 24th, 2021

DOI: https://doi.org/10.21203/rs.3.rs-748009/v1

License: @ (i) This work is licensed under a Creative Commons Attribution 4.0 International License. Read Full License 


\title{
Carryover of a saddle-node bifurcation after transforming a parameter into a variable
}

\author{
Carlos Contreras • Gustavo Carrero . \\ Gerda de Vries
}

Received: date / Accepted: date

\begin{abstract}
In this article, we introduce and study the carryover of a saddlenode bifurcation, a concept that describes how a saddle-node bifurcation of a dynamical system is carried over into an extended dynamical system obtained by transforming one of the parameters of the original system into a variable. We show that additional transversality and singularity conditions are needed to guarantee the carryover of a saddle-node bifurcation and provide a graphical methodology with a two-parameter bifurcation diagram to verify that such conditions are met. The results are applied to a gene activation model when the parameter describing the signal for activation is transformed into a variable, and to a cell cycle regulatory model when the parameter describing the cell mass is transformed into a variable. In both cases, we show that a saddle-node bifurcation carryover takes place.
\end{abstract}

Keywords Bifurcation · saddle-node bifurcation · bifurcation diagram · parameter transformation $\cdot$ mathematical modeling

Carlos Contreras

Collaborative Mathematical Biology Group (formerly Centre for Mathematical Biology), Department of Mathematical and Statistical Sciences, University of Alberta, Edmonton, AB T6G 2G1, Canada

E-mail: carlos.contreras@ualberta.ca

http://orcid.org/0000-0001-7928-607X

Gustavo Carrero

Centre for Science, Faculty of Science and Technology, Athabasca University, Edmonton, AB T5J 3S8, Canada

E-mail: gustavoc@athabasca.ca

Gerda de Vries

Collaborative Mathematical Biology Group (formerly Centre for Mathematical Biology), Department of Mathematical and Statistical Sciences, University of Alberta, Edmonton, AB T6G 2G1, Canada

E-mail: gerda.devries@ualberta.ca 


\section{Introduction}

Consider a dynamical system

$$
\dot{z}=f\left(z ; \mu_{1}, \mu_{2}\right),
$$

where $z \in \mathbb{R}^{n}$ and one of the parameters $\mu_{1}, \mu_{2} \in \mathbb{R}$ drives a saddle-node bifurcation as it crosses a critical value. Now, transforming one of the parameters, for example, $\mu_{1}$, into a variable, we obtain

$$
\begin{aligned}
\dot{z} & =f\left(z, \mu_{1} ; \mu_{2}\right), \\
\dot{\mu}_{1} & =g\left(\mu_{1} ; \mu_{2}\right),
\end{aligned}
$$

where $\left(z, \mu_{1}\right) \in \mathbb{R}^{n+1}$ and $g\left(\mu_{1} ; \mu_{2}\right)$ is the vector field of the new variable $\mu_{1}$. We are interested in the conditions under which a saddle-node bifurcation in the extended system occurs as $\mu_{2}$ varies. If the bifurcation point in the extended system, (2), is inherited (in a sense that will be clarified later) from the bifurcation point in the original system, (1), then we say that the bifurcation point in the extended system is the carryover of the bifurcation in the original system. We are choosing the word carryover to express the fact that the bifurcation can also be transferred to a new parameter that was not originally the bifurcation parameter, but other choices include inheritance, persistence, extension and continuation.

The main objective of this work is to find conditions that guarantee the carryover of a saddle-node bifurcation. Moreover, we would like to unfold the relationship, if any, between the saddle-node bifurcation in the original system and the saddle-node bifurcation in the extended system.

Our work is motivated by the study of saddle-node bifurcations in the mathematical models of biological systems. These saddle-node bifurcations often are associated with biologically meaningful properties such as biological switches and hysteresis. When adding more complexity to the model of a biological system exhibiting a saddle-node bifurcation, it might be important to preserve the existence of the bifurcation. In particular, if a set of differential equations describing a biological system (original system) exhibits a saddlenode bifurcation driven by a parameter, it might be relevant to add more complexity to the system by transforming the bifurcation parameter into a dynamical variable and obtain a system with one additional dimension (extended system). Note that in the extended system, a saddle-node bifurcation might still be present but with respect to another parameter. If this is the case, we call this property the carryover of a saddle-node bifurcation.

To illustrate the applicability of our work, we introduce the following two examples, which will be fully analyzed in Section 5. First, consider the dimensionless model for the activation of gene $x$ by a biochemical signal substance $s$ given by

$$
\dot{x}=s-r x+\frac{x^{2}}{1+x^{2}}
$$


where $r>0$ is the degradation rate, and $s \geq 0$ Strogatz 1994, Lewis et al. 1977). This model is characterized by an irreversible switch-like activation (critical transition) of gene $x$ as $s$ increases from zero above a threshold $s^{*}$ : This switch is driven by a saddle-node bifurcation at $s^{*}$ (see Section 5 for more details). The biochemical signal needs to be sufficiently high to increase the activity of the gene from low to high. Suppose that we include the dynamics of the substance $s$ by adding a differential equation for $s$ in the original system (3). We would be interested in finding the conditions that guarantee the carryover of the saddle-node bifurcation for the extended system with respect to the parameter $r$. In other words, we would like to know if the activation or inactivation of the gene $x$ could be driven now by the degradation rate $r$.

As a second example, we consider the regulatory network for the cell cycle involving the mutual antagonism of Cyclin B and APC (Anaphase Promoting Complex) that regulates the beginning and the end of the cell cycle. The beginning of the cell cycle, during the G1 phase, is characterized by low levels of Cyclin B caused by the action of active APC. Cell growth promotes APC inactivation, which in turn allows for Cyclin B synthesis in a critical transition, known as the start of the cell cycle. During the S-G2-M phase, Cyclin B activity is high which promotes activity of Cdc20, an activating subunit of APC. At the end of the S-G2-M phase, active APC lowers the level of Cyclin B, thus closing the cycle and defining the finish of the cell cycle. In this regulatory network, the cell mass acts as a parameter that drives the critical transition towards the beginning of the cell cycle through a saddle-node bifurcation in which the activity of Cyclin B increases quickly as the cell mass increases. The system describing the cell cycle is modelled by the following system of differential equations

$$
\begin{aligned}
& \frac{d Y}{d t}=k_{1}-\left(k_{2 p}+k_{2 p p} P\right) Y, \\
& \frac{d P}{d t}=\frac{\left(k_{3 p}+k_{3 p p} A\right)(1-P)}{J_{3}+(1-P)}-k_{4} m \frac{Y P}{J_{4}+P}, \\
& \frac{d A}{d t}=k_{5 p}+k_{5 p p} \frac{\left(m Y / J_{5}\right)^{n}}{1+\left(m Y / J_{5}\right)^{n}}-k_{6} A,
\end{aligned}
$$

where $Y$ is the concentration of Cyclin B, $P$ is the concentration of active APC (given by subunit Cdh1), $A$ is the concentration of active of Cdc20, $m$ is the cell mass, $k$ parameters are production and decay rates, and $J$ parameters are saturation constants, and $n$ is the Hill coefficient (Segel and Edelstein-Keshet 2013; Tyson and Novák 2001). This basic model for the cell cycle accounts for the G1 (or start) checkpoint, identified with an equilibrium where the concentration of Cyclin B is low, and the irreversible transition to S-G2-M phase, driven by a saddle-node bifurcation where the low level equilibrium of Cyclin B is lost (see Section 5 for more details).

This model can be extended by including more elements of the cell cycle, such as other Cyclin proteins and checkpoints (Tyson et al. 2002, 2003), or by considering the mass, $m$, as a variable (Contreras et al. |2019). In this latter 
case, we would have an extended system where the beginning of the cell cycle occurs dynamically as regulated by the G1 checkpoint, described by the saddlenode bifurcation with respect to the parameter $m$ in system (4). This leads to the question of whether or not there exists another parameter that drives the G1 checkpoint in the extended system through a saddle-node bifurcation. In other words, our interest would be to find the conditions that guarantee the carryover of the saddle-node bifurcation for the extended system with respect to a new parameter.

Note that in both examples, we transform the parameter that drives a saddle-node bifurcation into a variable, and aim to find the conditions for the extended system that guarantee the carryover of the saddle-node bifurcation so that the particular biological characteristics of the original system are preserved in the extended system.

In this paper, we provide sufficient conditions to guarantee the carryover of the saddle-node bifurcation going from the original system (1) to the extended system (2). For this purpose, we review the basic concepts of a saddle-node bifurcation in Section 2, state the main results for the carryover of a saddle-node bifurcation for the one-dimensional case in Section 3, and extend the results to the $n$-dimensional case in Section 4 . We provide a practical graphical approach that can be used to guarantee the carryover of a saddle-node bifurcation. In Section 5, we will apply the results to the two examples introduced above. Finally, in Section 6, we discuss the limitations of our results and future work.

\section{Mathematical Background}

Saddle-node bifurcations in $\mathbb{R}^{n}$ are characterized by three conditions: singularity, nondegeneracy, and transversality conditions. They guarantee the creation (or destruction) of two equilibria as one parameter crosses the bifurcation value. This is summarized in the following results taken from Meiss (2007. Ch. 8).

Theorem 1 (saddle node) Let $f \in C^{2}\left(\mathbb{R}^{n} \times \mathbb{R}^{k}, \mathbb{R}^{n}\right)$, and suppose that $f(z ; \mu)$ satisfies

$$
f(0 ; 0)=0, \quad \operatorname{spec}\left(D_{z} f(0 ; 0)\right)=\left\{0, \lambda_{2}, \lambda_{3}, \ldots, \lambda_{n}: \lambda_{k} \neq 0, k \neq 1\right\} .
$$

Choose coordinates so that $D_{z} f(0 ; 0)$ is diagonal in the zero eigenvalue and set $z=(x, y)$ where $x \in \mathbb{R}^{1}$ corresponds to the zero eigenvalue and $y \in \mathbb{R}^{n-1}$ are the remaining coordinates. Then

$$
\begin{aligned}
& \dot{x}=f_{1}(x, y ; \mu), \\
& \dot{y}=M y+f_{2}(x, y ; \mu),
\end{aligned}
$$

where $f_{1}(0,0 ; 0)=0, f_{2}(0,0 ; 0)=0, D_{z} f_{1}(0,0 ; 0)=0, D_{z} f_{2}(0,0 ; 0)=0$, and $M$ is an invertible matrix. Suppose that 


$$
D_{x x} f_{1}(0,0 ; 0)=c \neq 0 .
$$

Then there exists an interval $I(\mu)$ containing 0 , functions $y=\eta(x ; \mu)$ and extremal value $m(\mu)=\operatorname{Ext}_{x \in I(\mu)}\left[f_{1}(x ; \eta(\mu) ; \mu)\right]$, and a neighborhood of $\mu=0$ such that if $m(\mu) c>0$ there are no equilibria and if $m(\mu) c<0$ there are two. Suppose that $M$ has a $u$-dimensional unstable space and an $(n-u-1)$ dimensional stable space. Then, when there are two equilibria, one has a udimensional unstable manifold and an $(n-u)$-dimensional stable manifold and the other has a $(u+1)$-dimensional unstable manifold and an $(n-u-1)$ dimensional stable manifold.

Equations (5) and (7) are the singularity and nondegeneracy conditions, respectively. They are necessary conditions for the function $f_{1}$ to be zero up to the zero- and first-order approximations about the bifurcation point, but nonzero in the second-order approximation. The function $y=\eta(x ; \mu)$ allows us to reduce the dynamics in a neighborhood of the bifurcation point to onedimension, i.e.,

$$
\dot{x}=f_{1}(x, \eta(x ; \mu) ; \mu) .
$$

The extremal value function $m(\mu)$ determines a single condition on the parameters, $m(\mu)=0$, along which two equilibria are created (or destroyed). Having one condition on the parameters means that the bifurcation that takes place has codimension-one. In order to be a saddle-node bifurcation, the equilibria need to be created as some combination of the parameters crosses the bifurcation point. This can be guaranteed with a simple condition.

Corollary 1 If $\mu_{1}$ is a single parameter such that

$$
D_{\mu_{1}} f_{1}(0,0 ; 0) \neq 0
$$

then a saddle-node bifurcation takes place when $\mu_{1}$ crosses zero.

Equation (8) is known as the transversality condition because, geometrically, $m(\mu)=0$ is crossed transversally as $\mu_{1}$ crosses zero. Note that $\mu_{1}$ is an arbitrary parameter, and that the transversality condition can hold for several parameters at the same time. We only consider saddle-node bifurcations that take place as a single parameter crosses the bifurcation point.

In the context of this paper, we only consider two parameters for the sake of simplicity, i.e., $\mu=\left(\mu_{1}, \mu_{2}\right) \in \mathbb{R}^{2}$. We show that as long as a saddle-node bifurcation takes place in system (1) for at least one of the parameters, the extended system (2) also has a saddle-node bifurcation for the other parameter (i.e., the extended variable does not need to be a bifurcation parameter in the original system) under some singularity and transversality conditions.

The Implicit Function Theorem is an essential tool in the study of saddlenode bifurcations in general. For instance, the function $y=\eta(x ; \mu)$ in Theorem 1 is consequence of this theorem. The following form of the Implicit Function Theorem is taken from Meiss (2007, Ch. 8). 
Theorem 2 (implicit function) Let $U$ be an open set in $\mathbb{R}^{n} \times \mathbb{R}^{k}$ and $F \in C^{r}\left(U, \mathbb{R}^{n}\right)$ with $r \geq 1$. Suppose there is a point $\left(x_{0}, \mu_{0}\right) \in U$ such that $F\left(x_{0} ; \mu_{0}\right)=c$ and $D_{x} F\left(x_{0} ; \mu_{0}\right)$ is a nonsingular matrix. Then there are open sets $V \subset \mathbb{R}^{n}$ and $W \subset \mathbb{R}^{k}$ and a unique $C^{r}$ function $\xi(\mu): W \mapsto V$ for which $x_{0}=\xi\left(\mu_{0}\right)$ and $F(\xi(\mu) ; \mu)=c$.

\section{One-dimensional case}

In this section, we focus on the case where the variable $z$ in the system $(1)$ is one-dimensional, i.e., we consider the system

$$
\dot{x}=f\left(x ; \mu_{1}, \mu_{2}\right),
$$

where $x \in \mathbb{R}, \mu_{1}, \mu_{2} \in \mathbb{R}$, and $f$ is a sufficiently smooth function on $\left(x, \mu_{1}, \mu_{2}\right)$. This system is extended by transforming one of the parameters, for example, $\mu_{1}$, into a variable to define the extended system

$$
\begin{aligned}
\dot{x} & =f\left(x, \mu_{1} ; \mu_{2}\right), \\
\dot{\mu}_{1} & =g\left(\mu_{1} ; \mu_{2}\right),
\end{aligned}
$$

where $g\left(\mu_{1} ; \mu_{2}\right)$ is the sufficiently smooth vector field of the new variable $\mu_{1}$. We want to find conditions for the carryover of a saddle-node bifurcation in the original system $(9)$ to the extended system $(10)$.

Suppose, without loss of generality, that the saddle-node bifurcation for (9) occurs at the origin as one parameter, $\mu_{1}$, crosses zero. That is, $f$ at $\left(x, \mu_{1}, \mu_{2}\right)=(0,0,0)$ satisfies the singularity conditions

$$
\left\{\begin{array}{l}
f\left(x ; \mu_{1}, \mu_{2}\right)=0, \\
D_{x} f\left(x ; \mu_{1}, \mu_{2}\right)=0,
\end{array}\right.
$$

and the nondegeneracy and transversality conditions

$$
\left\{\begin{array}{l}
D_{x x} f\left(x ; \mu_{1}, \mu_{2}\right) \neq 0, \\
D_{\mu_{1}} f\left(x ; \mu_{1}, \mu_{2}\right) \neq 0 .
\end{array}\right.
$$

The left hand side of system (11) defines the function

$$
F\left(x, \mu_{1} ; \mu_{2}\right)=\left(\begin{array}{c}
f\left(x ; \mu_{1}, \mu_{2}\right) \\
D_{x} f\left(x ; \mu_{1}, \mu_{2}\right)
\end{array}\right),
$$

with Jacobian

$$
J=\left(\begin{array}{cc}
D_{x} f & D_{\mu_{1}} f \\
D_{x x} f & D_{x \mu_{1}} f
\end{array}\right)=\left(\begin{array}{cc}
0 & D_{\mu_{1}} f \\
D_{x x} f & D_{x \mu_{1}} f
\end{array}\right) .
$$

This matrix has full rank since

$$
\operatorname{det} J=\operatorname{det}\left(\begin{array}{cc}
0 & D_{\mu_{1}} f \\
D_{x x} f & D_{x \mu_{1}} f
\end{array}\right)=-D_{\mu_{1}} f D_{x x} f \neq 0
$$


by conditions (11) and (12). The Implicit Function Theorem 2 applied to $F$ guarantees the existence of an interval $I$ and unique functions

$$
\begin{aligned}
x & =\mathcal{X}\left(\mu_{2}\right), \\
\mu_{1} & =\mathcal{M}\left(\mu_{2}\right),
\end{aligned}
$$

for $\mu_{2} \in I$, such that

$$
\mathcal{X}(0)=0, \quad \mathcal{M}(0)=0,
$$

and the singularity conditions (11) are satisfied in $I$. This defines a smooth onedimensional curve $\Gamma$ that follows the bifurcation point $\left(x, \mu_{1}, \mu_{2}\right)=(0,0,0)$, and is parameterized by $\mu_{2}$, i.e.,

$$
\Gamma=\left\{\left(x, \mu_{1}, \mu_{2}\right): x=\mathcal{X}\left(\mu_{2}\right), \mu_{1}=\mathcal{M}\left(\mu_{2}\right), \mu_{2} \in I\right\},
$$

and satisfies (11).

By continuity, we can start from $\left(x, \mu_{1}, \mu_{2}\right)=(0,0,0)$ and follow the points that satisfy the singularity conditions (11) and the nondegeneracy and transversality conditions 12 to extend $\Gamma$. If the transversality condition is violated at some point, $D_{\mu_{1}} f=0$, but the same condition is satisfied for the other parameter, $D_{\mu_{2}} f \neq 0$, we apply similar arguments to parameterize $\Gamma$ by $\mu_{1}$ in that section. Hence, we can extend $\Gamma$ from the bifurcation point $\left(x, \mu_{1}, \mu_{2}\right)=(0,0,0)$ beyond the interval $I$ as long as the transversality condition is satisfied for at least one of the parameters (see Figure11). The projection of the extended $\Gamma$ onto the $\left(\mu_{1}, \mu_{2}\right)$-plane, given by $\pi:\left(x, \mu_{1}, \mu_{2}\right) \mapsto\left(\mu_{1}, \mu_{2}\right)$, defines an implicit function

$$
h\left(\mu_{1}, \mu_{2}\right)=0,
$$

known as bifurcation boundary, commonly plotted numerically using continuation (for example, using PyDSTool, XPPAUT, or MatCont). Note that although $\Gamma$ is a smooth curve, $h\left(\mu_{1}, \mu_{2}\right)=0$ is not necessarily smooth at every point. For more details on birfucation curves and two-parameter bifurcations see Kuznetsov (2004).

If the extended system (10) has a saddle-node bifurcation that is the carryover of the saddle-node bifurcation of interest in the original system (9), then this bifurcation must take place on $\Gamma$ as it is the set of points satisfying the conditions for a saddle-node bifurcation.

Proposition 1 Consider the system (9). Suppose $f\left(x ; \mu_{1}, \mu_{2}\right) \in C^{2}\left(\mathbb{R} \times \mathbb{R}^{2}, \mathbb{R}\right)$ with a nonhyperbolic equilibrium at the origin, $f(0 ; 0,0)=0, D_{x} f(0 ; 0,0)=0$, and satisfying the nondegeneracy condition

$$
D_{x x} f(0 ; 0,0) \neq 0,
$$

and transversality condition for either $\mu_{1}$ or $\mu_{2}$

$$
D_{\mu_{1}} f(0 ; 0,0) \neq 0 \quad \text { or } \quad D_{\mu_{2}} f(0 ; 0,0) \neq 0,
$$




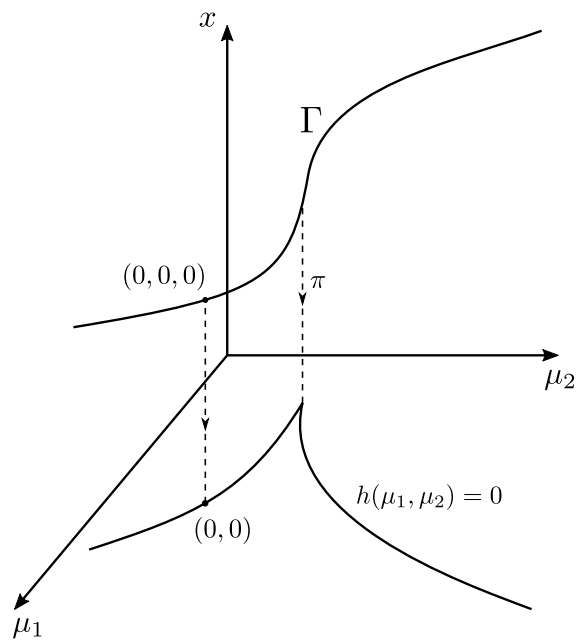

Fig. 1 A bifurcation curve $\Gamma$ and its corresponding bifurcation boundary $h\left(\mu_{1}, \mu_{2}\right)=0$ (projection onto the $\left(\mu_{1}, \mu_{2}\right)$-plane). Modified from Figure 8.1 in Kuznetsov 2004).

i.e., the system (9) has a saddle-node bifurcation where either $\mu_{1}$ or $\mu_{2}$ is the bifurcation parameter. This defines a one-dimensional smooth curve $\Gamma \subset \mathbb{R}^{3}$ in a neighbourhood of $\left(x, \mu_{1}, \mu_{2}\right)=(0,0,0)$ in which $f$ satisfies the singularity and nondegeneracy conditions.

Consider the extended system (10) by transforming parameter $\mu_{1}$ into a variable, where $f \in C^{2}\left(\mathbb{R}^{2} \times \mathbb{R}, \mathbb{R}\right)$ and $g \in C^{2}(\mathbb{R} \times \mathbb{R}, \mathbb{R})$. If there is a point $\left(x, \mu_{1}, \mu_{2}\right)=\left(x^{*}, \mu_{1}^{*}, \mu_{2}^{*}\right) \in \Gamma$ such that $g\left(\mu_{1} ; \mu_{2}\right)$ satisfies the singularity conditions

$$
g\left(\mu_{1}^{*} ; \mu_{2}^{*}\right)=0, \quad D_{\mu_{1}} g\left(\mu_{1}^{*} ; \mu_{2}^{*}\right)=b \neq 0,
$$

and the transversality condition

$$
\operatorname{det}\left(\begin{array}{ll}
D_{\mu_{1}} f & D_{\mu_{1}} g \\
D_{\mu_{2}} f & D_{\mu_{2}} g
\end{array}\right)=D_{\mu_{1}} f D_{\mu_{2}} g-D_{\mu_{1}} g D_{\mu_{2}} f \neq 0,
$$

at $\left(x, \mu_{1}, \mu_{2}\right)=\left(x^{*}, \mu_{1}^{*}, \mu_{2}^{*}\right)$, then the extended system 10 has a saddle-node bifurcation at $\left(x, \mu_{1}\right)=\left(x^{*}, \mu_{1}^{*}\right)$ as $\mu_{2}$ crosses $\mu_{2}^{*}$.

Moreover, there exists a unique function $\mu_{1}=\nu\left(\mu_{2}\right)$ such that $\mu_{1}^{*}=\nu\left(\mu_{2}^{*}\right)$, and the extended system is reduced to one dimension around $\left(x^{*}, \mu_{1}^{*}\right)$

$$
\dot{\xi}=f\left(\xi+\frac{a}{b}\left(\nu\left(\mu_{2}\right)-\mu_{1}^{*}\right)+x^{*}, \nu\left(\mu_{2}\right) ; \mu_{2}\right)-\frac{a}{b} g\left(\nu\left(\mu_{2}\right) ; \mu_{2}\right),
$$

where $\xi=x-x^{*}-\frac{a}{b}\left(\mu_{1}-\mu_{1}^{*}\right)$ and $a=D_{\mu_{1}} f\left(x^{*}, \mu_{1}^{*} ; \mu_{2}^{*}\right)$.

Proof Let $z=\left(x, \mu_{1}\right)^{T}$ and $F\left(z ; \mu_{2}\right)=F\left(x, \mu_{1} ; \mu_{2}\right)=\left(f\left(x, \mu_{1} ; \mu_{2}\right), g\left(\mu_{1} ; \mu_{2}\right)\right)^{T}$. By definition of $\Gamma$, the singularity conditions $\left(f=0\right.$ and $\left.D_{x} f=0\right)$, the nondegeneracy condition $\left(D_{x x} f \neq 0\right)$, and one of the transversality conditions 
$\left(D_{\mu_{1}} f \neq 0\right.$ or $\left.D_{\mu_{2}} f \neq 0\right)$ are satisfied at the point $\left(z^{*}, \mu_{2}^{*}\right)=\left(x^{*}, \mu_{1}^{*}, \mu_{2}^{*}\right) \in \Gamma$. Since $g\left(\mu_{1}^{*} ; \mu_{2}^{*}\right)=0$, we also have $F\left(x^{*}, \mu_{1}^{*} ; \mu_{2}^{*}\right)=0$ (first singularity condition for $F$ ). The Jacobian of $F$ evaluated at $z^{*}$ is

$$
A=D_{z} F\left(x^{*}, \mu_{1}^{*} ; \mu_{2}^{*}\right)=\left.\left(\begin{array}{cc}
D_{x} f & D_{\mu_{1}} f \\
D_{x} g & D_{\mu_{1}} g
\end{array}\right)\right|_{z=z^{*}}=\left(\begin{array}{ll}
0 & a \\
0 & b
\end{array}\right),
$$

where $a=D_{\mu_{1}} f\left(x^{*}, \mu_{1}^{*} ; \mu_{2}^{*}\right)$ and $b=D_{\mu_{1}} g\left(\mu_{1}^{*} ; \mu_{2}^{*}\right) \neq 0$, by assumption 15. Since $\operatorname{det}(A)=0$ and $\operatorname{tr}(A)=b \neq 0$, the eigenvalues of $A$ are $\lambda_{1}=0$ and $\lambda_{2}=b \neq 0$ with corresponding eigenvectors

$$
v_{\lambda_{1}}=\left(\begin{array}{l}
1 \\
0
\end{array}\right), \quad v_{\lambda_{2}}=\left(\begin{array}{l}
a \\
b
\end{array}\right) .
$$

Note that condition $D_{\mu_{1}} g \neq 0$ is needed to guarantee only one zero eigenvalue. Thus, $D_{z} F$ is singular with only one zero eigenvalue (second singularity condition for $F$ ).

The diagonalization matrix $P$ and its inverse are given by

$$
P=\left(\begin{array}{ll}
1 & a \\
0 & b
\end{array}\right), \quad P^{-1}=\frac{1}{b}\left(\begin{array}{cc}
b & -a \\
0 & 1
\end{array}\right) .
$$

Let the new shifted coordinates be defined by

$$
\left(\begin{array}{l}
\xi \\
v
\end{array}\right)=P^{-1}\left(\begin{array}{c}
x-x^{*} \\
\mu_{1}-\mu_{1}^{*}
\end{array}\right)=\left(\begin{array}{c}
x-x^{*}-\frac{a}{b}\left(\mu_{1}-\mu_{1}^{*}\right) \\
\frac{1}{b}\left(\mu_{1}-\mu_{1}^{*}\right)
\end{array}\right) .
$$

Then the corresponding extended system is given by

$$
\begin{aligned}
& \dot{\xi}=f\left(\xi+a v+x^{*}, b v+\mu_{1}^{*} ; \mu_{2}\right)-\frac{a}{b} g\left(b v+\mu_{1}^{*} ; \mu_{2}\right), \\
& \dot{v}=\frac{1}{b} g\left(b v+\mu_{1}^{*} ; \mu_{2}\right) .
\end{aligned}
$$

Define

$$
f_{1}\left(\xi, v ; \mu_{2}\right)=f\left(\xi+a v+x^{*}, b v+\mu_{1}^{*} ; \mu_{2}\right)-\frac{a}{b} g\left(b v+\mu_{1}^{*} ; \mu_{2}\right),
$$

and define $f_{2}\left(v ; \mu_{2}\right)$ such that

$$
\frac{1}{b} g\left(b v+\mu_{1}^{*} ; \mu_{2}\right)=b v+f_{2}\left(v ; \mu_{2}\right) .
$$

Then,

$$
\begin{aligned}
& \dot{\xi}=f_{1}\left(\xi, v ; \mu_{2}\right), \\
& \dot{v}=b v+f_{2}\left(v ; \mu_{2}\right) .
\end{aligned}
$$

Note that the singularity conditions are satisfied by construction,

$$
\begin{aligned}
& f_{1}\left(0,0 ; \mu_{2}^{*}\right)=f_{2}\left(0 ; \mu_{2}^{*}\right)=0, \\
& D_{\xi} f_{1}\left(0,0 ; \mu_{2}^{*}\right)=D_{v} f_{1}\left(0,0 ; \mu_{2}^{*}\right)=D_{\xi} f_{2}\left(0 ; \mu_{2}^{*}\right)=D_{v} f_{2}\left(0 ; \mu_{2}^{*}\right)=0 .
\end{aligned}
$$

The nondegeneracy condition for $f_{1}$ is satisfied since 


$$
\left.D_{\xi \xi} f_{1}\left(0,0 ; \mu_{2}^{*}\right)=D_{x x} f\left(x^{*}, \mu_{1}^{*} ; \mu_{2}^{*}\right)\right) \neq 0 .
$$

The transversality condition for $f_{1}$ follows from dividing the determinant in 16 by $-b \neq 0$ and the definition of $f_{1}$ (18)

$$
\begin{aligned}
& \left.\stackrel{\left(D_{\mu_{1}} f\left(x^{*}, \mu_{1}^{*} ; \mu_{2}^{*}\right)\right.}{a} D_{\mu_{2}} g\left(\mu_{1}^{*} ; \mu_{2}^{*}\right)-\underline{D}_{\mu_{1}} g\left(\mu_{1}^{*} ; \mu_{2}\right) D_{\mu_{2}} f\left(x^{*}, \mu_{1}^{*} ; \mu_{2}^{*}\right)\right) \neq 0, \\
& \Longrightarrow D_{\mu_{2}} f\left(x^{*}, \mu_{1}^{*} ; \mu_{2}^{*}\right)-\frac{a}{b} D_{\mu_{2}} g\left(\mu_{1}^{*} ; \mu_{2}^{*}\right)=D_{\mu_{2}} f_{1}\left(0,0 ; \mu_{2}^{*}\right) \neq 0 .
\end{aligned}
$$

Then, by Theorem 1 and Corollary 1 . the transformed system 19 has a saddle-node bifurcation point at $(0,0)$ as $\mu_{2}$ crosses $\mu_{2}^{*}$.

Transforming back to the variable $z$, we have that the extended system (10) has a saddle-node bifurcation point at $\left(x^{*}, \mu_{1}^{*}\right)$ as $\mu_{2}$ crosses $\mu_{2}^{*}$.

Now, denote

$$
F_{2}\left(v ; \mu_{2}\right)=b v+f_{2}\left(v ; \mu_{2}\right) .
$$

Note that $F_{2}\left(0 ; \mu_{2}^{*}\right)=0$, and $D_{v} F_{2}\left(0 ; \mu_{2}^{*}\right)=b \neq 0$ by equation 20$)$. By the Implicit Function Theorem 2, there is a neighbourhood of $\left(v, \mu_{2}\right)=\left(0, \mu_{2}^{*}\right)$ where there exists a unique function $v=\hat{\nu}\left(\mu_{2}\right)$ such that $\hat{\nu}\left(\mu_{2}^{*}\right)=0, F_{2}\left(\hat{\nu}\left(\mu_{2}\right) ; \mu_{2}\right)=$ 0 , and equation $(19)$ reduces to

$$
\dot{\xi}=f_{1}\left(\xi, \hat{\nu}\left(\mu_{2}\right) ; \mu_{2}\right) .
$$

Keeping in mind that $v=\frac{1}{b}\left(\mu_{1}-\mu_{1}^{*}\right)$ then

$$
\mu_{1}=b v+\mu_{1}^{*}=b \hat{\nu}\left(\mu_{2}\right)+\mu_{1}^{*} .
$$

Define $\nu\left(\mu_{2}\right)=b \hat{\nu}\left(\mu_{2}\right)+\mu_{1}^{*}$, then $\nu\left(\mu_{2}^{*}\right)=b \hat{\nu}\left(\mu_{2}^{*}\right)+\mu_{1}^{*}=\mu_{1}^{*}$. Finally, using the definition of $f_{1}\left(\xi, \nu ; \mu_{2}\right)$, we have

$$
\begin{aligned}
\dot{\xi} & =f_{1}\left(\xi, \hat{\nu}\left(\mu_{2}\right) ; \mu_{2}\right) \\
& =f_{1}\left(\xi, \frac{1}{b}\left(\nu\left(\mu_{2}\right)-\mu_{1}^{*}\right) ; \mu_{2}\right) \\
& =f\left(\xi+\frac{a}{b}\left(\nu\left(\mu_{2}\right)-\mu_{1}^{*}\right)+x^{*}, \nu\left(\mu_{2}\right) ; \mu_{2}\right)-\frac{a}{b} g\left(\nu\left(\mu_{2}\right) ; \mu_{2}\right) .
\end{aligned}
$$

This theorem provides a way to extend the scalar system (9) where a saddlenode occurs by transforming any parameter, $\mu_{1}$ for convenience, into a variable to obtain the extended system (2) where a saddle-node bifurcation now occurs as the other parameter, $\mu_{2}$, passes through some bifurcation value $\mu_{2}^{*}$. Thus, we say that the saddle-node bifurcation in the extended system is the carryover of the saddle-node bifurcation in the original system. Note that the transformed parameter, $\mu_{1}$, does not need to be the original bifurcation parameter. Also note that Proposition 1 requires that $g\left(\mu_{1} ; \mu_{2}\right)$ does not depend explicitly on $x$. This makes the conditions of this proposition easy to verify with graphical and numerical tools. 
Proposition 2 Under the conditions of Proposition (1), let $h\left(\mu_{1}, \mu_{2}\right)=0$ be the projection of $\Gamma$ onto the $\left(\mu_{1}, \mu_{2}\right)$-plane. If $h\left(\mu_{1}, \mu_{2}\right)$ is differentiable at $\left(\mu_{1}^{*}, \mu_{2}^{*}\right)$, then conditions (16) and 15) are equivalent to

1. $g\left(\mu_{1} ; \mu_{2}\right)=0$ intersects $h\left(\mu_{1}, \mu_{2}\right)=0$ transversally at a point $\left(\mu_{1}^{*}, \mu_{2}^{*}\right)$, and

2. the tangent line to $g\left(\mu_{1} ; \mu_{2}\right)=0$ at $\left(\mu_{1}^{*}, \mu_{2}^{*}\right)$ is not parallel to the $\mu_{1}$-axis,

respectively.

This proposition says that in order to find the saddle-node bifurcation points for the extended system, we plot the two-parameter bifurcation diagram of the original system, superimpose the nullclines of the new equation in the extended system, and look for transverse intersections between the saddle-node bifurcation curve and the nullclines. This is enough to verify the singularity and transversality conditions in the extended system.

Proof (Proof of Proposition 2) Two vectors $u, v \in \mathbb{R}^{2}$ are transverse (not parallel) if and only if the determinant of the matrix formed by them is nonzero (is zero), i.e.,

$$
\operatorname{det}(u, v)=u_{1} v_{2}-v_{1} u_{2}=|u||v| \sin (\theta) \neq 0 \Longleftrightarrow \theta \neq 0, \pi .
$$

Recall that $h\left(\mu_{1}, \mu_{2}\right)=0$ is defined by the projection of $\Gamma$ onto the $\left(\mu_{1}, \mu_{2}\right)$ plane, given by $\left(x, \mu_{1}, \mu_{2},\right) \mapsto\left(\mu_{1}, \mu_{2}\right)$. Since at least one of $D_{\mu_{1}} f$ or $D_{\mu_{2}} f$ is non-zero, points on $\Gamma$ have a unique correspondence to points on $h\left(\mu_{1}, \mu_{2}\right)=0$. Thus, a point $\left(\mu_{1}^{*}, \mu_{2}^{*}\right)$ at which $g=0$ and $h=0$ intersect has a unique corresponding point $\left(z^{*}, \mu_{2}^{*}\right)=\left(x^{*}, \mu_{1}^{*}, \mu_{2}^{*}\right) \in \Gamma$.

Assume $D_{\mu_{1}} f \neq 0$ at $\left(z^{*} ; \mu_{2}^{*}\right)$. Then, by the Implicit Function Theorem 2 we can parameterize $\Gamma$ by $\mu_{2}$ with functions $x=\mathcal{X}\left(\mu_{2}\right)$ and $\mu_{1}=\mathcal{M}\left(\mu_{2}\right)$ such that $x^{*}=\mathcal{X}\left(\mu_{2}^{*}\right)$ and $\mu_{1}^{*}=\mathcal{M}\left(\mu_{2}^{*}\right)$ (see equation (13)). Implicit differentiation of $f\left(x, \mu_{1} ; \mu_{2}\right)=0$ with respect to $\mu_{2}$ gives

$$
D_{x} f \mathcal{X}^{\prime}+D_{\mu_{1}} f \mathcal{M}^{\prime}+D_{\mu_{2}} f=0 .
$$

At $z^{*}, D_{x} f=0$ and we have

$$
\mathcal{M}^{\prime}=-\frac{D_{\mu_{2}} f}{D_{\mu_{1}} f} .
$$

Implicit differentiation of $h\left(\mu_{1}, \mu_{2}\right)=0$ with respect to $\mu_{2}$ gives

$$
D_{\mu_{1}} h \mathcal{M}^{\prime}+D_{\mu_{2}} h=0 .
$$

Evaluating at $z^{*}$, substituting the $\mathcal{M}^{\prime}$ and multiplying by $-D_{\mu_{1}} f$, we obtain

$$
D_{\mu_{1}} h D_{\mu_{2}} f-D_{\mu_{2}} h D_{\mu_{1}} f=0 .
$$

This means that vectors $\left(D_{\mu_{1}} f, D_{\mu_{2}} f\right)^{T}$ and $\left(D_{\mu_{1}} h, D_{\mu_{2}} h\right)^{T}$ are multiple of each other at $z^{*}$. Note that this is also true if $D_{\mu_{1}} f=0$ since we must have $D_{\mu_{2}} f \neq 0$ and similar arguments follow. Thus, $\left(D_{\mu_{1}} h, D_{\mu_{2}} h\right)^{T}$ and $\left(D_{\mu_{1}} g, D_{\mu_{2}} g\right)^{T}$ 
are transverse if and only if $\left(D_{\mu_{1}} f, D_{\mu_{2}} f\right)^{T}$ and $\left(D_{\mu_{1}} g, D_{\mu_{2}} g\right)^{T}$ are transverse, which is equivalent to saying that the transversality condition (16) holds.

Finally, the condition that the tangent line of $g\left(\mu_{1} ; \mu_{2}\right)=0$ at $\left(\mu_{1}^{*}, \mu_{2}^{*}\right)$ is not parallel to the $\mu_{1}$-axis is clearly equivalent to $D_{\mu_{1}} g\left(\mu_{1}^{*}, \mu_{2}^{*}\right) \neq 0$.

In the previous propositions, it is possible to generalize the arguments of the new scalar field, $g\left(\mu_{1} ; \mu_{2}\right)$, to include dependence on $x$, i.e., $g\left(x, \mu_{1} ; \mu_{2}\right)$, provided $\Gamma$ and $g=0$ intersect in the $\left(x, \mu_{1}, \mu_{2}\right)$-space. However, in the case of $g\left(\mu_{1} ; \mu_{2}\right)$, the conditions of Proposition 1 are easy to verify with the graphical and numerical tools given by Proposition 2 .

In order to illustrate the application of Propositions 1 and 2 , we introduce the following examples, where we consider a one-dimensional system with two parameters, $\mu_{1}$ and $\mu_{2}$,

$$
\dot{x}=f\left(x ; \mu_{1}, \mu_{2}\right),
$$

and transform the parameter $\mu_{1}$ into a variable to obtain the extended system

$$
\begin{aligned}
\dot{x} & =f\left(x, \mu_{1} ; \mu_{2}\right), \\
\dot{\mu}_{1} & =g\left(\mu_{1} ; \mu_{2}\right) .
\end{aligned}
$$

Example 1 Consider $f\left(x ; \mu_{1}, \mu_{2}\right)=-\mu_{1}-\mu_{2}-x^{2}$. Since $D_{x} f=-2 x=0$ at $x=0, D_{x x} f=-2 \neq 0$, and $D_{\mu_{1}} f=-1 \neq 0$, there is a saddle-node bifurcation at $x=0$ as $\mu_{1}$ crosses zero and $\mu_{2}=0$. Furthermore, since $D_{\mu_{2}} f=-1 \neq$ $0, \mu_{2}$ could be also taken as the bifurcation parameter when $\mu_{1}=0$. The bifurcation boundary is given by $h\left(\mu_{1}, \mu_{2}\right)=-\mu_{1}-\mu_{2}=0$. Figure 2 a shows the two-parameter bifurcation diagram along with the following three choices for $g\left(\mu_{1} ; \mu_{2}\right)$.

1. If $g\left(\mu_{1} ; \mu_{2}\right)=\mu_{1}+\mu_{2}$, then $g=0$ overlaps $h=0$ and they are never transverse. In fact, the extended system does not have a saddle-node bifurcation since it always has a unique steady state at $\left(x, \mu_{1}\right)=\left(0,-\mu_{2}\right)$, for each values of $\mu_{2}$.

2. If $g\left(\mu_{1} ; \mu_{2}\right)=-\mu_{1}+\mu_{2}$, then $g=0$ intersects $h=0$ transversally at $\left(\mu_{1}, \mu_{2}\right)=(0,0)$. According to Proposition 2, the extended system has a saddle-node bifurcation at $\left(x, \mu_{1}\right)=(0,0)$ as $\mu_{2}$ crosses $\mu_{2}=0$. In fact, there are two steady states, $\left(x, \mu_{1}\right)=\left( \pm \sqrt{-2 \mu_{2}}, \mu_{2}\right)$ when $\mu_{2}<0$, and they collide and disappear as $\mu_{2}$ becomes positive. Figure $2 \mathrm{~b}$ shows the bifurcation diagram for the extended system.

If we were interested in reducing the extended system to saddle-node bifurcation normal form, we could apply the second part of Proposition 1 to obtain the one-dimensional form (17) of the extended system. From the proof we see that the function $\mu_{1}=\nu\left(\mu_{2}\right)$ is obtained by solving $g=-\mu_{1}+\mu_{2}=0$, which gives $\mu_{1}=\nu\left(\mu_{2}\right)=\mu_{2}$. Thus, the extended system reduced to one-dimension around $\left(x, \mu_{1}\right)=(0,0)$ as $\mu_{2}$ crosses $\mu_{2}=0$ is

$$
\dot{\xi}=-\left(\xi-\mu_{2}\right)^{2}-2 \mu_{2},
$$

where $\xi=x+\mu_{1}$. 
3. If $g\left(\mu_{1} ; \mu_{2}\right)=\mu_{1}^{2}-\mu_{2}$, then $g=0$ intersects $h=0$ transversally at $\left(\mu_{1}, \mu_{2}\right)=(0,0)$ and $\left(\mu_{1}, \mu_{2}\right)=(-1,1)$, but the tangent line of $g\left(\mu_{1} ; \mu_{2}\right)=$ 0 at $\left(\mu_{1}, \mu_{2}\right)=(0,0)$ is parallel to the $\mu_{1}$-axis. Proposition 2 guarantees the saddle-node bifurcation at $\left(x, \mu_{1}, \mu_{2}\right)=(0,-1,1)$, but not at $\left(x, \mu_{1}, \mu_{2}\right)=(0,0,0)$. In fact, at $\left(x, \mu_{1}, \mu_{2}\right)=(0,0,0)$, there is a BogdanovTakens (double-zero) bifurcation (see Section 8.4 in Kuznetsov (2004)), since $D_{\mu_{1}} g=0$ implies that there are two zero eigenvalues. When $\mu_{2}=0$, there is a single steady state at $\left(x, \mu_{1}\right)=(0,0)$. When $0<\mu_{2}<1$, two steady states emerge from the origin, a stable node $\left(x, \mu_{1}\right)=\left(\sqrt{\sqrt{\mu_{2}}-\mu_{2}},-\sqrt{\mu_{2}}\right)$, and saddle $\left(x, \mu_{1}\right)=\left(-\sqrt{\sqrt{\mu_{2}}-\mu_{2}},-\sqrt{\mu_{2}}\right)$. When $\mu_{2}=1$ there is a saddle-node bifurcation at $\left(x, \mu_{1}\right)=(0,-1)$ as the two steady states collide and $D_{\mu_{1}} g \neq 0$. Figure 2 s shows the bifurcation diagram for the extended system.

To reduce the system to saddle-node normal form we solve $g=\mu_{1}^{2}-\mu_{2}=0$ for $\mu_{1}$. We take $\mu_{1}=\nu\left(\mu_{2}\right)=-\sqrt{\mu_{2}}$ since it passes through the point $\left(\mu_{1}, \mu_{2}\right)=(-1,1)$. Thus, the reduction of the extended system to onedimension around $\left(x, \mu_{1}\right)=(0,-1)$ as $\mu_{2}$ crosses $\mu_{2}=1$ is

$$
\dot{\xi}=-\left(\xi-\sqrt{\mu_{2}}+1\right)^{2}+\sqrt{\mu_{2}}\left(-\sqrt{\mu_{2}}+1\right),
$$

where $\xi=x-\mu_{1}-1$. To derive this normal form we found functions $f_{2}$, $F_{2}, \hat{\nu}$, and $\nu$ satisfying $F_{2}\left(\hat{\nu}\left(\mu_{2}\right) ; \mu_{2}\right)=0$, as defined within the proof of Proposition 1 (details are left as exercise for the interested reader). Note that this reduction is not possible around the Bogdanov-Takens bifurcation at $\left(x, \mu_{1}, \mu_{2}\right)=(0,0,0)$, since $\mu_{1}=\nu\left(\mu_{2}\right)$ is not defined in an open neighbourhood of $\left(\mu_{1}, \mu_{2}\right)=(0,0)$.

Example 2 Consider $f\left(x ; \mu_{1}, \mu_{2}\right)=\mu_{2}^{2}+1-\mu_{1}-x^{2}$. Since $D_{x} f=-2 x=0$ at $x=0, D_{x x} f=-2 \neq 0$, and $D_{\mu_{1}} f=-1 \neq 0$, there is a saddle-node bifurcation at $x=0$ as $\mu_{1}$ crosses $\mu_{1}=1$ and $\mu_{2}=0$. However, since $D_{\mu_{2}} f=2 \mu_{2}=0$ at $\mu_{2}=0$, there is no saddle-node bifurcation at $\left(x, \mu_{1}, \mu_{2}\right)=(0,1,0)$ if $\mu_{2}$ is taken as bifurcation parameter. The bifurcation boundary is given by $h\left(\mu_{1}, \mu_{2}\right)=\mu_{2}^{2}+1-\mu_{1}=0$. Figure 3 a shows the two-parameter bifurcation diagram along with the following three choices for $g\left(\mu_{1} ; \mu_{2}\right)$.

1. If $g\left(\mu_{1} ; \mu_{2}\right)=-\mu_{1}+2$, then $g=0$ intersects $h=0$ transversally twice, at $\left(\mu_{1}, \mu_{2}\right)=(2, \pm 1)$. Thus, by Proposition 2 , the extended system undergoes two saddle-node bifurcations at $\left(x, \mu_{1}\right)=(0,2)$, one as $\mu_{2}$ crosses $\mu_{2}=-1$ from the left where the two steady states, $\left(x, \mu_{1}\right)=\left( \pm \sqrt{\mu_{2}^{2}-1}, 2\right)$, collide and disappear, and one as $\mu_{2}$ crosses $\mu_{2}=1$ from the left where the two steady states, $\left(x, \mu_{1}\right)=\left( \pm \sqrt{\mu_{2}^{2}-1}, 2\right)$, emerge. Figure $3 p$ shows the bifurcation diagram for the extended system.

2. If $g\left(\mu_{1} ; \mu_{2}\right)=-\mu_{1}+1$, then $g=0$ is tangential to $h=0$ at $\left(\mu_{1}, \mu_{2}\right)=(1,0)$. No saddle-node bifurcation occurs since the two steady states $\left(x, \mu_{1}\right)=$ $\left( \pm \sqrt{\mu_{2}^{2}}, 1\right)=\left( \pm\left|\mu_{2}\right|, 1\right)$ collide and bounce back, as seen in Figure 3 c. In fact, at $\left(x, \mu_{1}, \mu_{2}\right)=(0,1,0)$, the extended system satisfies the singularity conditions $(\lambda=0,-1)$ and nondegeneracy condition $\left(D_{x x} f=-2 \neq 0\right)$, but 
a)

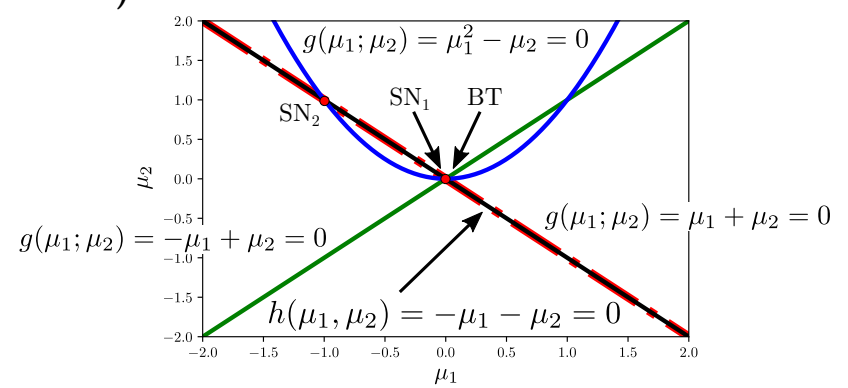

b)

c)
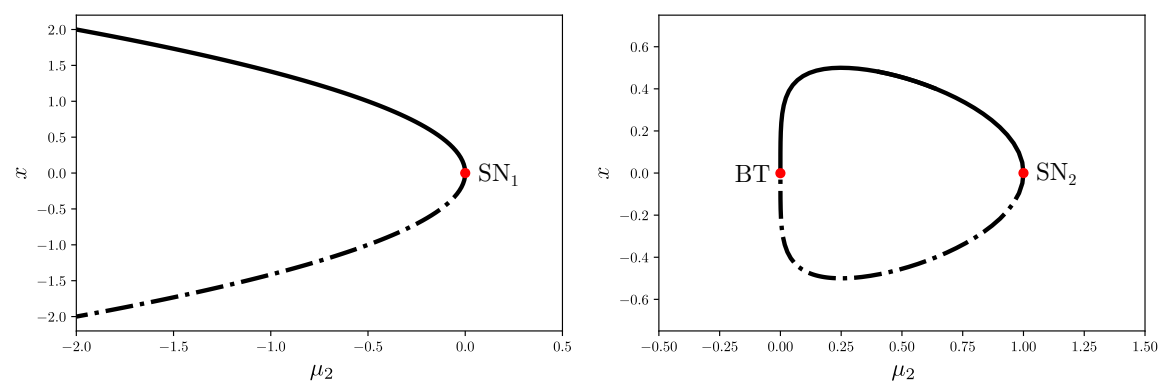

Fig. 2 Bifurcation diagrams for Example 1 where $f\left(x ; \mu_{1}, \mu_{2}\right)=-\mu_{1}-\mu_{2}-x^{2}$. a) Twoparameter bifurcation boundary (red dash-dotted line) for $f$ with $\mu_{1}$ and $\mu_{2}$ as bifurcation parameters, and nullclines for three choices of $g\left(\mu_{1} ; \mu_{2}\right): g\left(\mu_{1} ; \mu_{2}\right)=\mu_{1}+\mu_{2}$ (black) nullcline is never transverse; $g\left(\mu_{1} ; \mu_{2}\right)=-\mu_{1}+\mu_{2}$ (green) nullcline is transverse at $\left(\mu_{1}, \mu_{2}\right)=$ $(0,0)$; and $g\left(\mu_{1} ; \mu_{2}\right)=\mu_{1}^{2}+\mu_{2}$ (blue) nullcline is transverse at $\left(\mu_{1}, \mu_{2}\right)=(-1,1)$ and $\left(\mu_{1}, \mu_{2}\right)=(0,0)$ but $D_{\mu_{1}} g(0 ; 0)=0$. b) Bifurcation diagram when $g\left(\mu_{1} ; \mu_{2}\right)=-\mu_{1}+\mu_{2}$. A saddle-node bifurcation $\left(\mathrm{SN}_{1}\right)$ occurs at $\left(x, \mu_{1}, \mu_{2}\right)=(0,0,0)$. c) Bifurcation diagram when $g\left(\mu_{1} ; \mu_{2}\right)=\mu_{1}^{2}-\mu_{2}$. A saddle-node bifurcation $\left(\mathrm{SN}_{2}\right)$ occurs at $\left(x, \mu_{1}, \mu_{2}\right)=(0,1,1)$ and a Bogdanov-Takens (double-zero) bifurcation (BT) occurs at $\left(x, \mu_{1}, \mu_{2}\right)=(0,0,0)$.

not the transversality condition $\left(D_{\mu_{1}} f D_{\mu_{2}} g-D_{\mu_{1}} g D_{\mu_{2}} f=-\left.2 \mu_{2}\right|_{\mu_{2}=0}=\right.$ $0)$. Note that this is not a transcritical bifurcation since the steady states $\left(\left|\mu_{2}\right|, 1\right)$ and $\left(-\left|\mu_{2}\right|, 1\right)$ are a stable node (two negative eigenvalues) and a saddle point (eigenvalues with opposite sign), respectively, for all $\mu_{2}$. In other words, they do not exchange stability when they collide, instead they touch and bounce back preserving their stability.

By reducing the system to one-dimensional form, $\dot{\xi}=f_{1}\left(\xi ; \mu_{2}\right)=\mu_{2}^{2}-\xi^{2}$ (obtained by applying equation (17) with $\nu\left(\mu_{2}\right)=1$ ), where $\xi=x-$ $\mu_{1}+1$, we verify that this is not a saddle-node bifurcation, since it does not satisfy the transversality condition $D_{\mu_{2}} f_{1}=-2 \mu_{2}=0$ at $\mu_{2}=0$, nor a transcritical bifurcation, since it does not satisfy the transversality condition $D_{\xi \mu_{2}} f_{1}=-2 \mu_{2}=0$ at $\mu_{2}=0$,

3. If $g\left(\mu_{1} ; \mu_{2}\right)=\mu_{2}-\mu_{1}+1$, then $g\left(\mu_{1} ; \mu_{2}\right)=0$ is transverse at $\left(\mu_{1}, \mu_{2}\right)=(1,0)$ and $\left(\mu_{1}, \mu_{2}\right)=(2,1)$. Moreover, the tangent line to $g\left(\mu_{1}, \mu_{2}\right)=0$ at $(1,0)$ and $(2,1)$ is not parallel to the $\mu_{1}$-axis since $D_{\mu_{1}} g\left(\mu_{1} ; \mu_{2}\right)=-1$. Thus, as 
in the first case $\left(g\left(\mu_{1} ; \mu_{2}\right)=-\mu_{1}+2\right)$, two saddle-node bifurcations occur, one as $\mu_{2}$ crosses $\mu_{2}=0$ from the left where two steady states $\left(x, \mu_{1}\right)=$ $\left( \pm \sqrt{\mu_{2}\left(\mu_{2}-1\right)}, \mu_{2}+1\right)$ collide and disappear, and one as $\mu_{2}$ crosses $\mu_{2}=1$ from the left where two steady states $\left(x, \mu_{1}\right)=\left( \pm \sqrt{\mu_{2}\left(\mu_{2}-1\right)}, \mu_{2}+1\right)$ emerge. Figure $3 \mathrm{~d}$ shows the bifurcation diagram for the extended system. This case is interesting because at $\left(\mu_{1}, \mu_{2}\right)=(1,0)$, the transversality condition is not satisfied for the original system with respect to $\mu_{2}$, i.e., $D_{\mu_{2}} f(0 ; 1,0)=0$. In other words, even if $\mu_{2}$ is not a bifurcation parameter in the original system at $\left(x^{*}, \mu^{*}\right), \mu_{2}$ becomes a bifurcation parameter in the extended system at the same point.

4. If we transform the parameter $\mu_{2}$ into a variable, instead of $\mu_{1}$, using $\dot{\mu}_{2}=$ $g\left(\mu_{2} ; \mu_{1}\right)=\mu_{2}-\mu_{1}+1$, it follows from the previous case that two saddlenode bifurcations occur at steady states $\left(x, \mu_{2}\right)=(0,0)$ and $\left(x, \mu_{2}\right)=$ $(0,1)$ as $\mu_{1}$ crosses $\mu_{1}=1$ and $\mu_{1}=2$, respectively, as seen in Figure 3 . However, note that even though $\mu_{2}$ is not a bifurcation parameter in the original system at $\left(x, \mu_{1}, \mu_{2}\right)=(0,1,0)$ there is a carryover of the saddlenode bifurcation that occurs in the original system (with $\mu_{1}$ as bifurcation parameter) when transforming $\mu_{2}$ into a variable.

Propositions 1 and 2 will be used in Section 5 in the analysis of the first biological application, namely the gene activation model (3).

\section{$4 n$-dimensional case}

In the previous section, we showed the carryover of a saddle-node bifurcation for the one-dimensional case. In this section, we show that this result also holds for the $n$-dimensional case. In short, this is true because the saddle-node bifurcations can be reduced to one-dimension around the bifurcation point.

Suppose that $f$ in the original system (1) satisfies the conditions of Theorem 1. Then we can reduce the system to the one-dimensional form

$$
\dot{x}=f_{1}\left(x, \eta\left(x ; \mu_{1}, \mu_{2}\right) ; \mu_{1}, \mu_{2}\right),
$$

in a neighbourhood of $(x, y, \mu)=(0,0,0)$ (in regards to the saddle-node bifurcation), where functions $f_{1}\left(x, y ; \mu_{1}, \mu_{2}\right)$ and $y=\eta\left(x ; \mu_{1}, \mu_{2}\right)$ are given by the theorem.

Now, assume that $f_{1}$ satisfies the transversality condition of Corollary 1 for either $\mu_{1}$ or $\mu_{2}$. Then, in a similar fashion as in the one-dimensional case, the Implicit Function Theorem 2 guarantees the existence of an interval $I$ and unique functions $\mathcal{X}$ and $\mathcal{M}$ such $x$ and $\mu_{1}$ can be parameterized in terms of $\mu_{2}$ (for example), i.e.,

$$
x=\mathcal{X}\left(\mu_{2}\right), \mu_{1}=\mathcal{M}\left(\mu_{2}\right), \quad \text { where } \quad \mu_{2} \in I, \quad \text { and } \quad \mathcal{X}(0)=0, \mathcal{M}(0)=0 .
$$

This defines the smooth one-dimensional bifurcation curve 
16

Contreras, Carrero, and de Vries

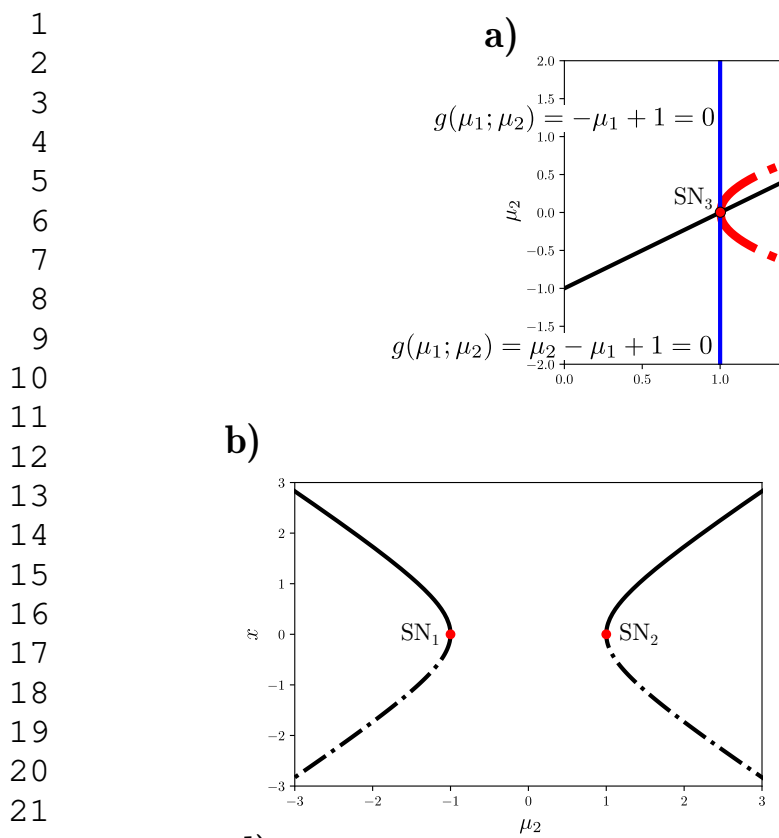

d)

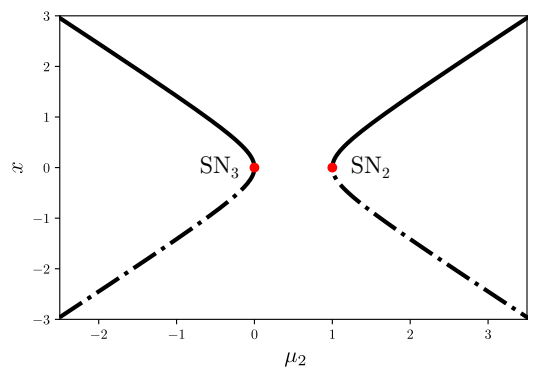

c)

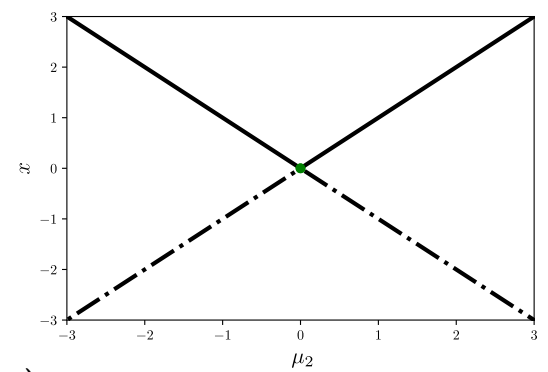

e)

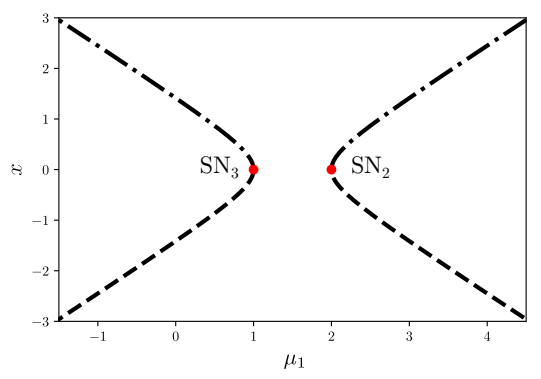

Fig. 3 Bifurcation diagrams for Example 2 where $f\left(x ; \mu_{1}, \mu_{2}\right)=\mu_{2}^{2}+1-\mu_{1}-x^{2}$. a) Twoparameter bifurcation boundary (red dash-dotted curve) for $f$ with $\mu_{1}$ and $\mu_{2}$ as bifurcation parameters, and nullclines for three choices of $g\left(\mu_{1} ; \mu_{2}\right): g\left(\mu_{1} ; \mu_{2}\right)=-\mu_{1}+2$ (green) nullcline is transverse at $\left(\mu_{1}, \mu_{2}\right)=(2,-1)$ and $\left(\mu_{1}, \mu_{2}\right)=(2,1) ; g\left(\mu_{1} ; \mu_{2}\right)=-\mu_{1}+1$ (blue) is tangential at $\left(\mu_{1}, \mu_{2}\right)=(1,0)$; and $g\left(\mu_{1} ; \mu_{2}\right)=\mu_{2}-\mu_{1}+1$ (black) nullcline is transverse at $\left(\mu_{1}, \mu_{2}\right)=(1,0)$ and $\left(\mu_{1}, \mu_{2}\right)=(2,1)$. b) Bifurcation diagram when $g\left(\mu_{1} ; \mu_{2}\right)=-\mu_{1}+2$. Two saddle-node bifurcations occur at $\left(x, \mu_{1}, \mu_{2}\right)=(0,2, \pm 1)$. c) Bifurcation diagram when $g\left(\mu_{1} ; \mu_{2}\right)=-\mu_{1}+1$. No saddle-node bifurcation occurs because the steady states collide but do not disappear. d) Bifurcation diagram when $g\left(\mu_{1} ; \mu_{2}\right)=\mu_{2}-\mu_{1}+1$. Two saddle-node bifurcations occur at $\left(x, \mu_{1}, \mu_{2}\right)=(0,1,0)$ and $\left(x, \mu_{1}, \mu_{2}\right)=(0,1,1)$. e) Bifurcation diagram when $\dot{\mu}_{2}=g\left(\mu_{2} ; \mu_{1}\right)=\mu_{2}-\mu_{1}+1$. Two saddle-node bifurcations occur at $\left(x, \mu_{2}\right)=(0,0)$ and $\left(x, \mu_{2}\right)=(0,1)$ as $\mu_{1}$ crosses $\mu_{1}=1$ and $\mu_{1}=2$, respectively. 


$$
\begin{aligned}
\Gamma=\left\{\left(z, \mu_{1}, \mu_{2}\right): z=(x, y)=\left(\mathcal{X}\left(\mu_{2}\right), \eta\left(\mathcal{X}\left(\mu_{2}\right)\right.\right.\right. & \left.\left.; \mathcal{M}\left(\mu_{2}\right), \mu_{2}\right)\right), \\
& \left.\mu_{1}=\mathcal{M}\left(\mu_{2}\right), \mu_{2} \in I\right\} .
\end{aligned}
$$

To extend $\Gamma$ in this case, we take a point in $\Gamma$, different from $(x, y, \mu)=$ $(0,0,0)$, and apply again Theorem 1 (after some appropriate translation) followed by the Implicit Function Theorem 2. Note that functions $f_{1}, \mathcal{X}$, and $\mathcal{M}$ do not need to be the same as before. By continuity, we can apply this process repetitively and further extend $\Gamma$ as long as the transversality condition holds for either $\mu_{1}$ and $\mu_{2}$ at each step. Finally, the bifurcation boundary, $h\left(\mu_{1}, \mu_{2}\right)=0$, is defined by the projection of the extended $\Gamma$ onto the $\left(\mu_{1}, \mu_{2}\right)$-plane, given by $\pi\left(z, \mu_{1}, \mu_{2}\right) \mapsto\left(\mu_{1}, \mu_{2}\right)$. Thus, the definition of $\Gamma$ and $h\left(\mu_{1}, \mu_{2}\right)=0$ is similar to the one-dimensional case.

Proposition 3 Let $f\left(z ; \mu_{1}, \mu_{2}\right) \in C^{2}\left(\mathbb{R}^{n} \times \mathbb{R}^{2}, \mathbb{R}^{n}\right)$ and suppose that the hypotheses of Theorem 1 are satisfied at $\left(z, \mu_{1}, \mu_{2}\right)=(0,0,0)$. Suppose also that the transversality condition in Corollary 1 is satisfied for either $\mu_{1}$ or $\mu_{2}$. Let $m\left(\mu_{1}, \mu_{2}\right)$ be the extremal value defined in Theorem 1. This defines a onedimensional smooth curve $\Gamma \subset \mathbb{R}^{n+2}$ in a neighbourhood of $\left(z, \mu_{1}, \mu_{2}\right)$ that satisfies the singularity and nondegeneracy conditions.

Consider the extended system (2) by transforming parameter $\mu_{1}$ into a variable, where $f \in C^{2}\left(\mathbb{R}^{n+1} \times \mathbb{R}, \mathbb{R}^{n}\right)$ and $g \in C^{2}(\mathbb{R} \times \mathbb{R}, \mathbb{R})$. If there is a point $\left(z, \mu_{1}, \mu_{2}\right)=\left(z^{*}, \mu_{1}^{*}, \mu_{2}^{*}\right) \in \Gamma$ such that $g\left(\mu_{1} ; \mu_{2}\right)$ satisfies

$$
g\left(\mu_{1}^{*} ; \mu_{2}^{*}\right)=0, \quad b=D_{\mu_{1}} g\left(\mu_{1}^{*} ; \mu_{2}^{*}\right) \neq 0,
$$

and the transversality condition

$$
\operatorname{det}\left(\begin{array}{cc}
D_{\mu_{1}} m & D_{\mu_{1}} g \\
D_{\mu_{2}} m & D_{\mu_{2}} g
\end{array}\right)=D_{\mu_{1}} h D_{\mu_{2}} g-D_{\mu_{1}} g D_{\mu_{2}} h \neq 0,
$$

is satisfied at $\left(z, \mu_{1}, \mu_{2}\right)=\left(z^{*}, \mu_{1}^{*}, \mu_{2}^{*}\right)$, then the extended system (2) has a saddle-node bifurcation at $\left(z, \mu_{1}\right)=\left(z^{*}, \mu_{1}^{*}\right)$ as $\mu_{2}$ crosses $\mu_{2}^{*}$.

Proof First, we translate the point $z^{*}$ to the origin using a new variable $\zeta=$ $z-z^{*}$ to obtain the translated system

$$
\dot{\zeta}=f\left(\zeta+z^{*} ; \mu_{1}, \mu_{2}\right)
$$

that satisfies all the conditions of Theorem 1 at $\zeta=0$. By Theorem 1 , we choose new translated coordinates $x \in \mathbb{R}$ and $y \in \mathbb{R}^{n-1}$ such that

$$
\begin{aligned}
& \dot{x}=f_{1}\left(x, y ; \mu_{1}, \mu_{2}\right), \\
& \dot{y}=M y+f_{2}\left(x, y ; \mu_{1}, \mu_{2}\right),
\end{aligned}
$$

where $f_{1}=0, f_{2}=0, D_{x} f_{1}=0, D_{x} f_{2}=0, D_{y} f_{1}=0, D_{y} f_{2}=0$, and $D_{x x} f_{1} \neq 0$ at $\left(x, y ; \mu_{1}, \mu_{2}\right)=\left(0,0 ; \mu_{1}^{*}, \mu_{2}^{*}\right)$, and $M$ is invertible. Moreover, 
there is an interval $I\left(\mu_{1}, \mu_{2}\right)$ of 0 and function $y=\eta\left(x ; \mu_{1}, \mu_{2}\right)$ where the extremal value

$$
m\left(\mu_{1}, \mu_{2}\right)=\operatorname{Ext}_{x \in I\left(\mu_{1}, \mu_{2}\right)}\left[f_{1}\left(x, \eta\left(x ; \mu_{1}, \mu_{2}\right) ; \mu_{1}, \mu_{2}\right)\right]
$$

is defined. Therefore, the extended system (2) can be reduced to

$$
\begin{aligned}
\dot{x} & =f_{3}\left(x, \mu_{1} ; \mu_{2}\right)=f_{1}\left(x, \eta\left(x ; \mu_{1}, \mu_{2}\right) ; \mu_{1}, \mu_{2}\right), \\
\dot{\mu}_{1} & =g\left(\mu_{1} ; \mu_{2}\right),
\end{aligned}
$$

in a neighborhood of $\left(\zeta, \mu_{1}, \mu_{2}\right)=\left(0, \mu_{1}^{*}, \mu_{2}^{*}\right)$ where the singularity and nondegeneracy conditions are satisfied.

By assumption, the transversality condition, $D_{\mu_{i}} f_{1} \neq 0$, is satisfied for either $\mu_{1}$ or $\mu_{2}$. Then, by Proposition 11, this system has a saddle-node bifurcation at $\left(x, \mu_{1}\right)=\left(0, \mu_{1}^{*}\right)$ as $\mu_{2}$ crosses $\mu_{2}^{*}$. It follows that the extended system (2) has a saddle-node bifurcation at $\left(z, \mu_{1}\right)=\left(0, \mu_{1}^{*}\right)$ as $\mu_{2}$ crosses $\mu_{2}^{*}$.

As we might expect, Proposition 2 also applies to the $n$-dimensional case. The proof follows similar arguments to the one-dimensional case.

Proposition 4 Under the conditions of Proposition 3. let $h\left(\mu_{1}, \mu_{2}\right)=0$ be the projection of $\Gamma$ onto the $\left(\mu_{1}, \mu_{2}\right)$-plane. If $h\left(\mu_{1}, \mu_{2}\right)$ is differentiable at $\left(\mu_{1}^{*}, \mu_{2}^{*}\right)$, then conditions (23) and 22 are equivalent to

1. $g\left(\mu_{1} ; \mu_{2}\right)=0$ intersects $h\left(\mu_{1}, \mu_{2}\right)=0$ transversally at a point $\left(\mu_{1}^{*}, \mu_{2}^{*}\right)$, and

2. the tangent line to $g\left(\mu_{1} ; \mu_{2}\right)=0$ at $\left(\mu_{1}^{*}, \mu_{2}^{*}\right)$ is not parallel to the $\mu_{1}$-axis,

respectively.

Example 3 Consider the system

$$
\begin{aligned}
& \dot{x}=\mu-x^{2}+x y-x y^{2}, \\
& \dot{y}=\lambda-y-x^{2}+x^{2} y,
\end{aligned}
$$

taken from Meiss (2007, p. 292). There is a saddle-node bifurcation at the origin as $\mu$ crosses zero when $\lambda=0$. The two-parameter bifurcation diagram starting from this bifurcation point is shown in Figure 4 . Now, consider the extended system

$$
\begin{aligned}
& \dot{x}=\mu-x^{2}+x y-x y^{2}, \\
& \dot{y}=\lambda-y-x^{2}+x^{2} y, \\
& \dot{\mu}=g(\mu ; \lambda)=\mu-\frac{1}{2} .
\end{aligned}
$$

The $\mu$-nullcline is transverse to the two-parameter bifurcation diagram in two points near $\lambda=0.5$ and $\lambda=1.1$ (see Figure 4 a). Since the tangent line of $g(\mu ; \lambda)$ is not parallel to the $\mu$-axis at any of the intersection points, we conclude, by Proposition 4, that there is a saddle-node carryover with $\lambda$ as the bifurcation parameter. The bifurcation diagrams for $x$ and $y$ are shown in 
a)

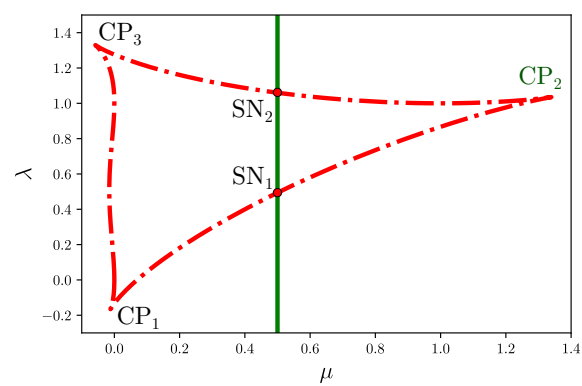

b)

c)
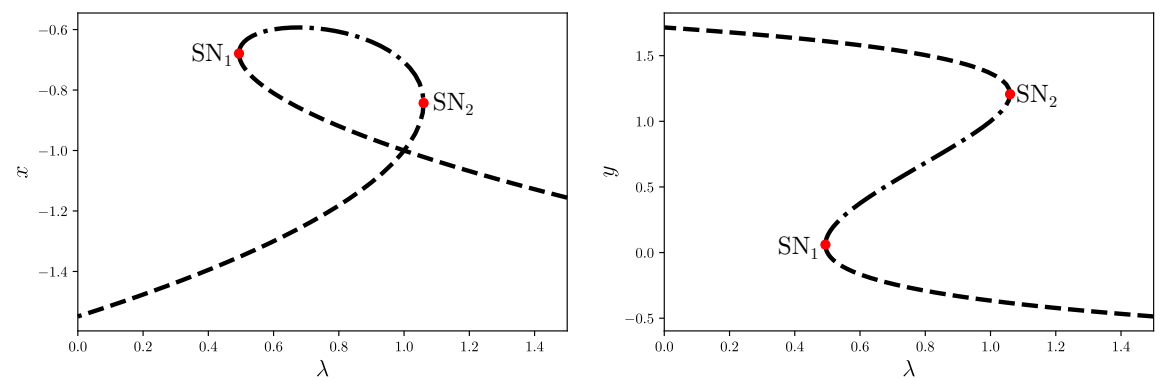

Fig. 4 Bifurcation diagrams for Example 3 a) Two-parameter bifurcation boundary (red dashed-dotted curve) and nullcline for $g(\mu ; \lambda)=\mu-\frac{1}{2}$ (green). Two saddle-node bifurcations $\left(\mathrm{SN}_{1}\right.$ and $\left.\mathrm{SN}_{2}\right)$ occur at the transverse intersection between the $g$-nullcline and the bifurcation boundary. Note that there are cusp bifurcations $\left(\mathrm{CP}_{1}, \mathrm{CP}_{2}\right.$, and $\left.\mathrm{CP}_{3}\right)$ when a pair of saddle-node bifurcation points collide. $\mathbf{b}-\mathbf{c}$ ) Bifurcation diagrams for the extended system with $\lambda$ as bifurcation parameter and variables $x$ and $y$ in the ordinate axis, respectively. The dashed lines indicate the unstable node with associated three-dimensional unstable manifold, while the dot-dashed lines indicate the saddle-node with associated one-dimensional stable manifold and two-dimensional unstable manifold.

Figures $4 \mathrm{p}$ and 4 , respectively, and the corresponding bifurcation points are found to be

$$
\begin{aligned}
& (x, y, \mu, \lambda) \approx(-0.6792,0.0604,0.5,0.4940), \\
& (x, y, \mu, \lambda) \approx(-0.8429,1.2069,0.5,1.0599) .
\end{aligned}
$$

\section{Applications}

In this section, we apply Propositions 1 and 2 to the gene activation model (3), and Proposition 4 to the cell cycle regulatory model (4). 
a)

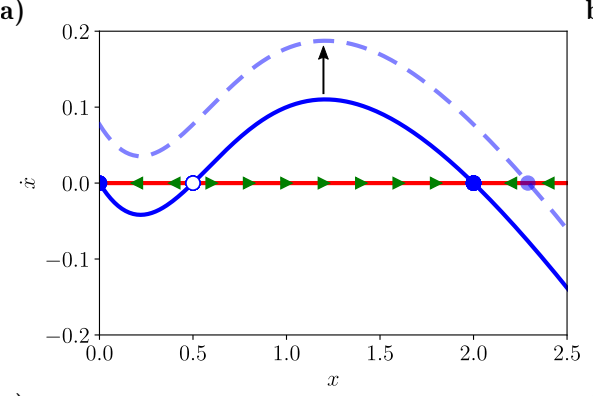

b)

c)

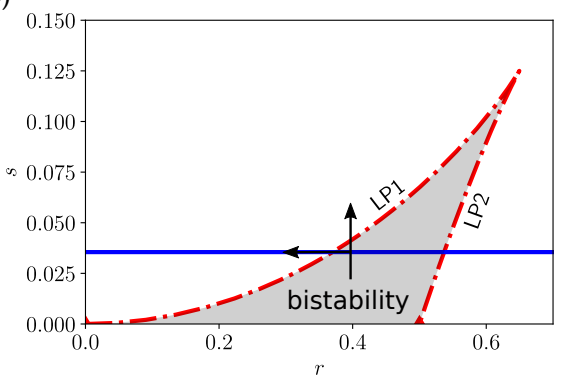

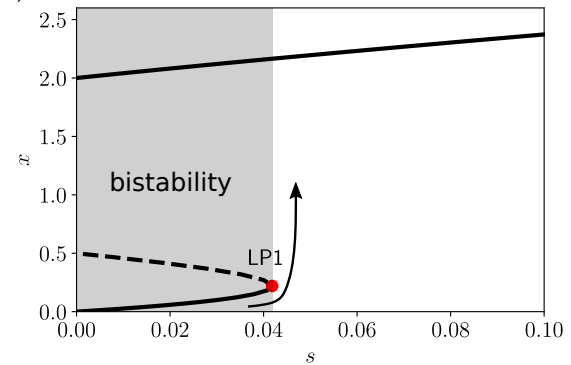

d)

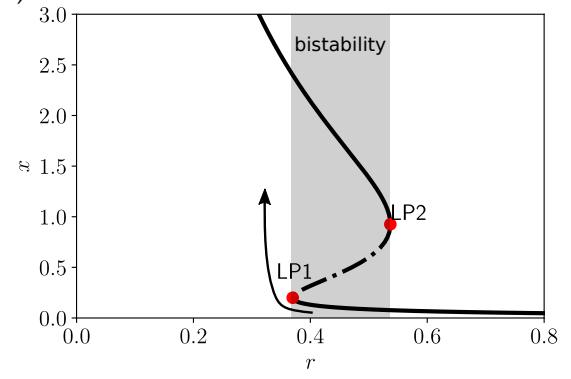

Fig. 5 a) Phase plot of 3 for $r=0.4$ and $s=0$ (solid) and $s=0.06$ (dashed). The critical transition occurs when $s$ increases and the left and middle equilibria collide and disappear at $s^{*} \approx 0.0418$, forcing gene activity to the remaining (stable) equilibrium to the right. b) Bifurcation diagram of the original system (3) using $s$ as bifurcation parameter. The arrow indicates gene activation and the saddle-node bifurcation point at $s^{*}$ is labeled as LP1. c) Two-parameter bifurcation diagram of $(3)$ with $r$ and $s$ as bifurcation parameters. A vertical cross section at $r=0.4$ corresponds to $\mathbf{b}$ and the vertical arrow matches that in $\mathbf{b}$. The bifurcation boundary (red curve) is the continuation of the point LP1 in $\mathbf{b}$ projected onto the $r s$-plane. The point in the upper right corner corresponds to $x=\sqrt{3} / 3$ (nondegeneracy condition not met) where a cusp bifurcation occurs. The blue horizontal line is the nullcline $g=0$ (for $c=\frac{a}{b}=\frac{6}{169}$ in 25 ) of the extended system, which intersects the bifurcation boundary transversally and Proposition 2 applies. d) Bifurcation diagram for the extended system (3) and (25) using $r$ as the bifurcation parameter. Switch activation of the gene is possible if $r$ is decreased below the bifurcation point labeled as LP1. The arrow matches the horizontal arrow in $\mathbf{c}$.

\subsection{Gene activation}

Consider the gene activation model (3), which is in the form of (1), with

$$
f(x ; r, s)=s-r x+\frac{x^{2}}{1+x^{2}} .
$$

For $r<\frac{1}{2}$, this model is characterized by the existence of a switch activation of the gene via a saddle-node bifurcation as $s$ increases from zero (see Figures 5 b). With initial condition $x(0)=0$, increasing $s$ would drive a critical transition that brings the activity of gene $x$ to a high value, thus activating the gene. However, if the value of $s$ is decreased, the activity of the gene remains in the active mode. For $r=0.4$ the bifurcation occurs at $s^{*} \approx 0.0418$. 
For this simple application, we can compute all conditions satisfying Theorem 1 and Corollary 1 From the singularity conditions, $f=0$ and $D_{x} f=0$, we obtain a parametrization of $r$ and $s$ in terms of $0<x \leq 1$,

$$
r=\frac{2 x}{\left(1+x^{2}\right)^{2}}, \quad s=\frac{x^{2}\left(1-x^{2}\right)}{\left(1+x^{2}\right)^{2}} .
$$

The nondegeneracy condition requires

$$
D_{x x} f=\frac{2\left(1-3 x^{2}\right)}{\left(1+x^{2}\right)^{3}} \neq 0 \Longrightarrow x \neq \frac{\sqrt{3}}{3},
$$

but the transversality conditions, $D_{s} f=1 \neq$ and $D_{r} f=-x \neq 0$, do not impose extra conditions. Thus, the bifurcation curve is given by

$$
\Gamma=\left\{(x, r, s): r=\frac{2 x}{\left(1+x^{2}\right)^{2}}, s=\frac{x^{2}\left(1-x^{2}\right)}{\left(1+x^{2}\right)^{2}}, x \in(0,1]\right\},
$$

and there is a saddle-node bifurcation at any $(x, a, b) \in \Gamma$ except for $x \neq \frac{\sqrt{3}}{3}$. In Figure 5, we show the projection of $\Gamma$ (bifurcation boundary) onto the $r s$-plane. Here we see that the bifurcation boundary separates the parameter space into two regions which differ in the number of equilibria, and that the switch activation of the gene via $s$ corresponds to crossing the upper bifurcation boundary from below.

Now consider transforming $s$ into a variable with constant synthesis and linear degradation terms

$$
\dot{s}=g(s ; r)=a-b s .
$$

where $a, b>0$. Note that the signal $s$ goes to a stable equilibrium $s=c=\frac{a}{b}$. If $c=\frac{6}{169} \approx 0.0355<s^{*} \approx 0.0418$, starting from initial conditions $x(0)=0$ and $s(0)=0$, the gene will not become active since the trajectory always remains within the bistability region (see Figure 5c). Would it be possible to activate the gene by varying the inactivation rate $r$ ? Note that the point $(x, s, r)=\left(\frac{1}{5}, \frac{6}{169}, \frac{125}{338}\right)$ belongs to $\Gamma$ and satisfies the singularity conditions (15), $g=0$ and $D_{s} g=-b \neq 0$; and transversality condition (16),

$$
\operatorname{det}\left(\begin{array}{ll}
D_{s} f & D_{s} g \\
D_{r} f & D_{r} g
\end{array}\right)=\left(\begin{array}{cc}
1 & -b \\
-x & 0
\end{array}\right)=-x b=-\frac{1}{5} b \neq 0 .
$$

Thus, by Proposition 1 there is a saddle-node bifurcation at $r^{*}=\frac{125}{338} \approx 0.3698$ as $r$ decreases from 0.4. In fact, this point was found by substituting $x=\frac{1}{5}$ into (24).

We can also apply Proposition 2 to the two-parameter bifurcation Figure 5;, where we observe that the nullcline $g=0$ crosses the bifurcation boundary transversally and the nullcline is not tangential to the $s$-axis at the intersection. In this figure, it is clear that the switch activation occurs in both the original and the extended systems as the corresponding bifurcation parameter crosses the upper bifurcation boundary from the bistability region into 
the region with only one equilibrium. This can be verified from the bifurcation diagram of the extended system as shown in Figure $5 \mathrm{~d}$. Therefore, the saddlenode bifurcation associated with the switch activation of the gene driven by $r$ in the extended system is the carryover of the saddle-node bifurcation associated with the switch gene activation of the gene driven by $s$ in the original system.

\subsection{Cell cycle start}

Consider the cell cycle model (4) where $z=(Y, P, A)$. This basic model is characterized by a saddle-node bifurcation driven by the cell mass, $m$, which is associated with the start of the cell cycle, as illustrated in Figure 6 6 . The lower stable equilibrium is associated with the G1 phase since the concentration of $Y$ is low. If $m$ remains lower than approximately $m^{*} \approx 0.8$, the cell remains in G1 checkpoint as the concentration of $Y$ needs to increase for the progression of the cell cycle. As $m$ increases beyond the bifurcation value $m^{*}$, the lower equilibrium is lost, allowing for the concentration of $Y$ to increase abruptly and the start of the cell cycle. It can be shown that this bifurcation is in fact a global saddle-node-loop bifurcation and the details of this can be found in Segel and Edelstein-Keshet (2013), but for the purpose of this paper we focus on the local saddle-node bifurcation.

We now include the dynamics of the slow parameter $m$ by transforming it into a variable following logistic growth

$$
\frac{d m}{d t}=\mu m\left(1-\frac{m}{K}\right),
$$

where $\mu$ is the growth rate, and $K$ is the carrying capacity. If $K$ is larger than the bifurcation value $\left(m^{*} \approx 0.8\right)$, then the start of the cell cycle occurs naturally as $m$ increases dynamically beyond $m^{*}$. We are interested in G1 checkpoint activation driven by $k_{5 p}$ as this parameter can be thought to be modulated by Polo-like kinase 1 (Plk1), a enzyme that plays a role in Cdc20 activation and has the potential to be used in cancer treatment through regulation of the cell cycle (Hansen et al. 2004; Liu et al. 2017). That is, we are interested in the carryover of the saddle-node bifurcation into the extended system with $k_{5 p}$ as the bifurcation parameter. Note that, as opposed to the previous application, computing the bifurcation curve $\Gamma$ in this example is considerably more complicated. We therefore study the potential carryover of the saddle-node bifurcation graphically by applying Proposition 4.

The two-parameter bifurcation diagram (using $m$ and $k_{5 p}$ as bifurcation parameters) for the original system (4) is shown in Figure 6p. The start of the cell cycle is seen here as crossing the lower portion of the bifurcation boundary from the G1 region to the right. With the addition of equation (26), the nullcline $g=0$ appears at $m=K$. If $K=2$, for example, there is a saddlenode bifurcation for $k_{5 p} \approx 0.025$ as the intersection between the nullcline and the bifurcation boundary is transversal and not parallel to the $m$-axis, and 
a)

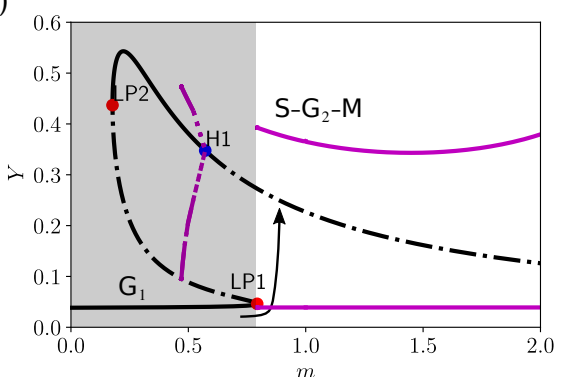

b)

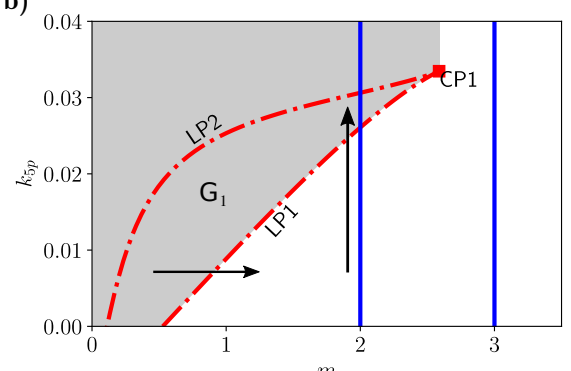

c)

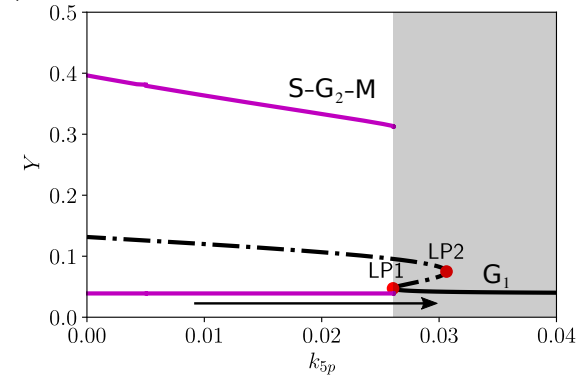

Fig. 6 a) Bifurcation diagram for the original system (4) with $m$ as the bifurcation parameter. The arrow indicates the transition from G1 to S-G2-M at the start of the cell cycle when the cell mass increases and the stable equilibrium of low $Y$ concentration disappears via a saddle-node bifurcation (labeled LP1). In the S-G2-M region, a limit cycle appears (globally, LP1 is a saddle-node-loop bifurcation), indicated in pink by maximum and minimum concentration of $Y$, in which the concentration of $\mathrm{Y}$ increases and decreases (the decrease in $Y$ concentration defines the finish of the cell cycle). b) Two-parameter bifurcation diagram for the original system (4) with $m$ and $k_{5 p}$ as bifurcation parameters. The horizontal arrow matches that in a indicating the start of the cell cycle. The red curve has two branches corresponding to the continuation of LP1 and LP2 in a. The nullcline $m=K$ of 26 is indicated in blue for $K=2$ and $K=3$. For $k=2$, the nullcline crosses the bifurcation curve transversally and Proposition 4 applies. For $k=3$, the nullcline does not cross the bifurcation curve and thus G1 checkpoint activation is not possible if varying $k_{5 p}$. c) Bifurcation diagram for extended system (4) and 26] with $k_{5 p}$ as the bifurcation parameter. The arrow indicates the G1 checkpoint activation, and matches the vertical arrow in $\mathbf{b}$.

Proposition 4 applies. Thus, we can increase $k_{5 p}$ beyond the intersection value (possibly with Plk1 treatment) to enter the G1 region and activate the G1 checkpoint, and allow it to drop down (possibly by natural degradation) in order to resume the cell cycle. However, if $K=3$, G1 checkpoint activation would not be possible by modifying $k_{5 p}$ as the saddle-node bifurcation is lost beyond $m \approx 2.6$ in a cusp bifurcation. The bifurcation diagram of the extended system using $k_{5 p}$ as bifurcation parameter is shown in Figure 6e, showing the appearance of a stable equilibrium associated with the G1 checkpoint where the concentration of $Y$ is low. 


\section{Discussion}

The carryover of a bifurcation is a concept that contributes to the development and analysis of mathematical models. In this paper, we studied the carryover of a saddle-node bifurcation; we take a system where a saddle-node bifurcation is present, extend it by transforming a parameter into a variable (adding one extra equation), and find the conditions that guarantee that a saddle-node bifurcation is still present. Our focus was restricted to the case where there are two parameters of interest and the vector field of the new equation does not depend on the variables already present in the original system. We showed that additional singularity and transversality conditions for the extended system are sufficient for the carryover of a saddle-node bifurcation on top of the necessary singularity, non-degeneracy, and transversality conditions for the original system. We provided a graphical approach to verify such conditions using a numerical continuation software, such as AUTO, XPP, MatCont, and PyDSTool.

We provided a proof of our statement for the one-dimensional case in Proposition 1, which was later used to prove the $n$-dimensional case in Proposition 3. Our proofs require the Implicit Function Theorem 2 to follow the bifurcation curve, and the Saddle Node Bifurcation Theorem 11 to either verify the conditions for a saddle-node bifurcation in the one-dimensional case or to reduce the system to one dimension at the saddle-node bifurcation for the $n$-dimensional case. Equivalent graphical results for the one-dimensional and $n$-dimensional cases are given in Propositions 2 and 4 respectively. These graphical results provide a practical numerical tool to study all the points where a carryover is possible based on a two-parameter bifurcation diagram and the nullclines of the new vector field.

We illustrate our results with two applications in mathematical biology where the carryover of a saddle-node bifurcation is of interest. The first application is the one-dimensional gene activation model, in which we are able to show the carryover of a saddle-node bifurcation both theoretically and numerically. The second application is a model for the cell cycle progression, in which we are only able to show the carryover numerically. In practice, the study of bifurcations is generally carried out numerically; thus, our graphical approach becomes a powerful tool to study the carryover of a saddle-node bifurcation in applications.

The development of the carryover of a saddle-node bifurcation was motivated by the results in Contreras et al. (2019), where the authors found a saddle-node bifurcation driven by radiation in a larger version of the cell cycle model with radiation pathway. Furthermore, in Contreras (2020), the author showed that this saddle-node bifurcation is the carryover of the saddle-node bifurcation driven by the mass in the cell cycle model studied by Tyson and Novák (2001). Other applications in which the carryover of a saddle-node bifurcation might take place include: variations of the cell cycle model (Tyson et al. 2001), a model of inflammatory response (Reynolds et al. 2006), a model of 
glucose and insulin levels in diabetes (Topp et al.|2000), and coupling-bursting models (De Vries and Sherman 2001).

Our result applies whether or not the parameter that is being transformed into a variable is the bifurcation parameter in the original system; we only need to start from a saddle-node bifurcation point and continue the bifurcation curve with respect to two parameters. Interestingly, a bifurcation for the extended system can take place at a point at which a bifurcation is not possible in the original system with respect to the same parameter because the transversality condition is not satisfied. Cases like this one are illustrated in this article with examples.

It is still left to study the generalization of the carryover. For example, the case where the bifurcation exists in the original system as both $\mu_{1}$ and $\mu_{2}$ change simultaneously (but not individually) or where $k$ parameters change simultaneously. The case where the new differential equation depends on the other variables, i.e., $\dot{\mu}_{1}=g\left(z, \mu_{1} ; \mu_{2}\right)$ in equation (2), is also not covered here. Assuming the bifurcation curve and the $\mu_{1}$-nullcline intersect in $\mathbb{R}^{n+2}$, an extra condition (or conditions) would be required to guarantee that the matrix $A$, as defined within the proof of Proposition (1), is invertible. We leave this case open for future research.

Our results open the interesting exploration of the carryover of other types of bifurcation (transcritical, pitchfork, Hopf, etc) and the idea of creating a different type of bifurcation when extending the system. We believe that studying the carryover of bifurcations from the theoretical and numerical perspective adds great value to the area of mathematical modelling.

Acknowledgements The authors would like to thank Dr. Thomas Hillen and Dr. John J. Tyson for their invaluable comments and suggestions towards the development of the carryover concept.

\section{References}

Contreras C (2020) Mathematical modelling of the effect of low-dose radiation on the $\mathrm{g} 2 / \mathrm{m}$ transition and the survival fraction via the atm/chk2 pathway. $\mathrm{PhD}$ thesis, University of Alberta

Contreras C, Carrero G, de Vries G (2019) A Mathematical Model for the Effect of Low-Dose Radiation on the G2/M Transition. Bulletin of Mathematical Biology

De Vries G, Sherman A (2001) From spikers to bursters via coupling: Help from heterogeneity. Bulletin of Mathematical Biology 63(2):371-391

Hansen DV, Loktev AV, Ban KH, Jackson PK (2004) Plk1 Regulates Activation of the Anaphase Promoting Complex by Phosphorylating and Triggering SCF $\beta$ TrCP -dependent Destruction of the APC Inhibitor Emi1. Molecular Biology of the Cell 15(12):5623-5634

Kuznetsov YA (2004) Elements Of Applied Bifurcation Theory. Springer

Lewis J, Slack J, Wolpert L (1977) Thresholds in development. Journal of Theoretical Biology 65(3):579-590

Liu Z, Sun Q, Wang X (2017) PLK1, A potential target for cancer therapy. Translational Oncology 10(1):22-32

Meiss JD (2007) Differential Dynamical Systems. SIAM 
Reynolds A, Rubin J, Clermont G, Day J, Vodovotz Y, Bard Ermentrout G (2006) A reduced mathematical model of the acute inflammatory response: I. Derivation of model and analysis of anti-inflammation. Journal of Theoretical Biology 242(1):220-236

Segel LA, Edelstein-Keshet L (2013) A Primer on Mathematical Models in Biology. SIAM Strogatz SH (1994) Nonlinear Dynamics and Chaos. Perseus Books

Topp B, Promislow K, Devries G, Miura RM, Finegood DT (2000) A model of $\beta$-cell mass, insulin, and glucose kinetics: Pathways to diabetes. Journal of Theoretical Biology 206(4):605-619

Tyson JJ, Novák B (2001) Regulation of the eukaryotic cell cycle: Molecular antagonism, hysteresis, and irreversible transitions. Journal of Theoretical Biology 210(2):249-263

Tyson JJ, Chen KC, Novák B (2001) Network dynamics and cell physiology. Nature reviews Molecular cell biology 2(12):908-916

Tyson JJ, Csikasz-Nagy A, Novák B (2002) The dynamics of cell cycle regulation. BioEssays 24(12):1095-1109

Tyson JJ, Chen KC, Novak B, Novák B (2003) Sniffers, buzzers, toggles and blinkers: Dynamics of regulatory and signaling pathways in the cell. Current Opinion in Cell Biology 15(2):221-231 